\title{
基于通信信号的水下目标主动探测 多普勒估计与补偿方法
}

\author{
卢俊，张群飞，史文涛，张玲玲
}

(西北工业大学 航海学院, 陕西 西安 710072)

\begin{abstract}
摘 要: 水下探测通信一体化利用通信发射信号进行目标主动探测, 而多普勒效应的存在使得主动探 测中目标参数估计性能恶化。为了消除多普勒效应的影响,提出一种基于频谱细化与校正的联合多 普勒估计和补偿方法。该方法利用通信发射信号中的同步头信号获取目标回波信号时延,截取信号 中的单频信号段。再利用离散傅里叶变换( DFT) 求得单频信号段最大幅值所对应的频率, 并在该频 率附近范围内进行频谱细化与校正求出频率偏移量, 从而估计出多普勒因子, 并根据所估计的多普勒 因子对接收的目标回波信号进行补偿。仿真结果表明,所提的频谱细化与校正联合多普勒估计与补 偿方法提高了多普勒估计精度, 使多普勒补偿后的信号与发射副本相关处理增益增加,提高了波达方 向(DOA) 估计性能, 并且对不同多普勒效应具有良好的鲁棒性。
\end{abstract}

\section{关 键 词: 多普勒估计与补偿; 探测通信一体化; 通信信号; 频谱细化; 频谱校正 \\ 中图分类号:TP393 \\ 文献标志码 : A}

水下探测通信一体化系统利用通信发射信号作 为共享信号, 在进行信息传输的同时实现目标主动 探测 ${ }^{[1]}$ 。相较于传统的主动声呐发射信号, 通信发 射信号具有持续时间长、发射声源级低的特点, 为了 提高一体化系统探测性能, 可利用发射副本与目标 回波进行互相关预处理。但由于目标和平台的相对 运动、水声声速低以及水声通信信号带宽较宽等因 素产生的多普勒效应严重 ${ }^{[2]}$, 导致回波发生时频域 扩展, 从而降低了互相关处理增益, 降低了目标参数 估计性能。为了解决这个问题, 需要对目标回波进 行多普勒估计与补偿。

空中多普勒估计被广泛应用于无线电、激光测 速等领域 ${ }^{[3-5]}$, 而水下多普勒估计在被应用于声学 多普勒测速仪 ${ }^{[6-7]}$ 的同时, 更为重要的应用是水声 通信, 因为多普勒效应恶化水声通信系统的码元同 步和载频跟踪, 导致误码率增加、接收性能下降 [8]。文献 $[9]$ 利用时域互相关估计法, 在发射信号
首尾插人对多普勒不敏感且相关性较强的信号, 利 用接收信号长度变化估计多普勒因子,但该算法受 限于数据帧的帧长。针对时域互相关估计的局限 性, 文献 $[10]$ 提出利用信号插值进行多普勒频移粗 估计, 然后采用信道均衡技术进一步消除多普勒效 应。文献 $[11]$ 以时域互相关估计进行粗估计, 再利 用固定频偏进行精补偿。文献 [12]利用相关器获 得信号的模糊函数, 再通过信号的相关性估计多普 勒频移。

上述方法都是在时域上进行, 而在频域上多普 勒估计更受关注。文献 [13]在发射信号前加人单 频信号, 在接收端利用快速傅里叶变换 (FFT) 得到 多普勒频率偏移量, 从而估计出多普勒因子。但是 该方法受限于频率分辨率, 针对该问题, 提出了对频 谱进行插值处理 ${ }^{[14]}$ 。文献 [15] 提出了基于牛顿插 值的多普勒估计增强技术, 文献 [16]在接收端进行 窄带滤波和 FFT 处理, 并利用抛物线插值拟合估计 
频率变化量。由于插值法对多普勒的估计精度有 限,因此提出了基于窄带谱的频谱细化分析方法,其 基本思想是对信号频谱中感兴趣的频谱进行局部放 大分析 ${ }^{[17]}$ 。常见的频谱细化方法有 Goertzel 算

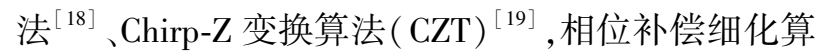
法 ${ }^{[20]}$ 以及 Zoom-FFT 法 ${ }^{[21]}$ 等, 理论上频谱能无限被 细化, 但随着细化倍数的增加, 计算复杂度也随之增 加。频谱校正是另一种频谱分析方法, 其对消除相 位、幅度与频率误差有较好的性能 ${ }^{[22]}$ 。并在实际工 程应用中, 频谱校正法具有良好的频率估计性能, 估 计误差小于频率分辨率 ${ }^{[23]}$ 。

针对水下探测通信一体化系统中主动探测性能 受多普勒效应影响的问题, 提出了基于频谱细化与 补偿的联合多普勒估计与补偿方法。首先利用同步 头信号获取回波到达时间, 截取回波中单频信号段。 然后, 对单频信号端加窗, 利用 DFT 获取最大幅值 对应的频率, 并在该频率附近利用 CZT 进行频谱细 化。随后, 对频谱进行校正, 得到最终的频率估计 值, 计算出多普勒因子。最后, 对回波信号进行多普 勒补偿, 并利用互相关多重信号分类 (MUSIC) 算法 进行 DOA 估计。仿真结果表明, 所提的频谱细化与 校正联合多普勒估计与补偿方法较其他算法具有更 精确、更稳定的估计性能, 且多普勒补偿后的 DOA 估计更为精确。

\section{1 系统模型}

\section{1 信号模型}

水下探测通信一体化系统工作示意图以及共享 信号帧结构如图 1 所示。一体化系统主要由发射 端、接收端以及电子仓三部分构成。发射端为单通 道溢流环换能器, 接收端为八元均匀线列阵, 电子仓 包含信号产生、发射、记录、处理等电路系统。通信 发射信号作为共享信号在实现通信信息传输的同

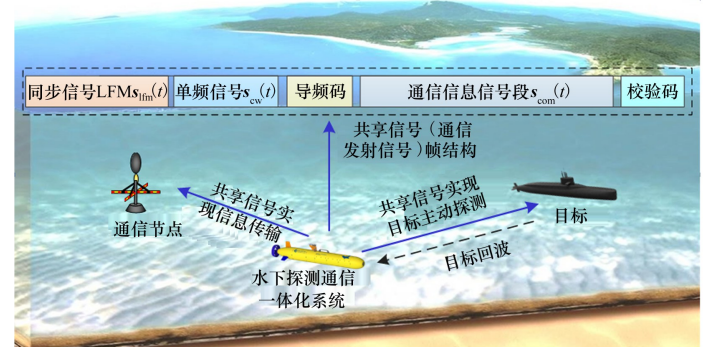

图 1 水下探测通信一体化工作示意图及共享信号帧结构
时, 作为主动探测信号实现目标主动探测。共享信 号 $s_{T}(t)$ 由 LFM 同步信号 $s_{\mathrm{lfm}}(t)$ 、频率为 $f_{0}$ 的单频信 号 $s_{\mathrm{cw}}(t)$ 、导频码、通信信息段 $s_{\mathrm{com}}(t)$ 以及校验码五 部分组成。

假设目标与一体化系统距离为 $R$, 水下声速为 $c$, 目标与一体化系统的相对径向速度为 $v$, 则目标 回波信号的声延时为

$$
\tau=\frac{2 R}{c}
$$

相应的多普勒扩展因子可表示为

$$
\alpha=\frac{2 v}{c}
$$

考虑信号衰减系数为 $a$, 则目标回波信号的时域表 达式为

$$
s_{R}(t)=a \cdot s_{T}[(1+\alpha)(t-\tau)]+n(t)
$$

式中, $n(t)$ 为零均值的高斯白噪声。

\section{2 互相关 MUSIC 波达方向估计}

假设有 $L$ 个远场目标, 方位角为 $\theta_{l}(l=1,2, \cdots$, $L)$, 接收阵为阵元数为 $M$ 的均匀线列阵, 阵元间距 $d=\lambda / 2, \lambda$ 为共享信号中通信信息信号段 $s_{\text {com }}(t)$ 载 频率对应的波长, 则阵列输出为

$$
\boldsymbol{X}(t)=\boldsymbol{A S}(t)+\boldsymbol{N}(t)
$$

式中: $\boldsymbol{X}(t)$ 为 $M \times N$ 维数据矩阵 ( $N$ 为快拍数); $S(t)$ 为 $L \times N$ 维目标信号矩阵; $N(t)$ 为 $M \times N$ 维加 性噪声矩阵; $\boldsymbol{A}=\left[\begin{array}{llll}\boldsymbol{a}\left(\theta_{1}\right) & \boldsymbol{a}\left(\theta_{2}\right) & \cdots & \boldsymbol{a}\left(\theta_{L}\right)\end{array}\right]$ 为 $M \times L$ 维阵列流型矢量矩阵, 阵列流型矢量 $\boldsymbol{a}\left(\theta_{L}\right)$ 为

$$
\boldsymbol{a}\left(\theta_{l}\right)=\left[\begin{array}{llll}
1 & \mathrm{e}^{-\mathrm{j} 2 \pi \sin \theta_{l} d / \lambda} & \cdots & \mathrm{e}^{-\mathrm{j} 2 \pi \sin \theta_{l}(M-1) d / \lambda}
\end{array}\right]
$$

发射信号 $\boldsymbol{s}_{T}(t)$ 与第 $m$ 个阵元接收数据 $\boldsymbol{X}_{m}(t)$ 作互 相关处理, 即有

$$
\boldsymbol{R}_{X_{m} s_{T}}\left(t_{0}\right)=\mathrm{E}\left[\boldsymbol{X}_{m}(t) \boldsymbol{s}_{T}^{*}\left(t-t_{0}\right)\right]
$$

则阵列接收信号与发射信号的互相关矩阵为

$$
\boldsymbol{R}_{X_{s_{T}}}=\left[\begin{array}{llll}
\boldsymbol{R}_{X_{1} s_{T}} & \boldsymbol{R}_{X_{2^{s} T}} & \cdots & \boldsymbol{R}_{X_{M^{s} T}}
\end{array}\right]
$$

利用 MUSIC 算法对目标 DOA 进行估计, 互相关矩 阵的协方差矩阵为

$$
\boldsymbol{R}=\boldsymbol{R}_{X_{s_{T}}} \boldsymbol{R}_{X_{s_{T}}}^{\mathrm{H}}
$$

对其进行特征值分解得到噪声子空间 $\boldsymbol{U}_{N}$, 即有互 相关 MUSIC 方法的谱估计表达式为

$$
\boldsymbol{P}_{\text {corr_music }}=\frac{1}{\boldsymbol{a}^{\mathrm{H}}(\theta) \boldsymbol{U}_{N} \boldsymbol{U}_{N}^{\mathrm{H}} \boldsymbol{a}(\theta)}
$$

通过谱峰搜索, 找出 $L$ 个最大值对应的角度即 为目标所对应的方位角。互相关处理提高接收信号 
的信噪比,从而提高了 MUSIC 算法 DOA 估计性能。 但是由于多普勒效应导致目标回波拉伸或压缩, 副 本与接收信号互相关产生失配, 降低参数估计精度。 为了解决多普勒效应导致副本与接收信号互相关失 配问题,提出了基于频谱细化与校正联合多普勒估 计与补偿方法。

\section{2 频谱细化与校正联合多普勒估计与 补偿}

频谱细化与校正联合多普勒估计与补偿主要分 为两步:第一步找出接收信号单频信号段幅值最大 频率点, 并在频率点附近进行频谱细化; 第二步对细 化的频谱进行校正并完成多普勒精估计及补偿。

\section{1 初步频谱细化}

假设接收阵列各个阵元受到多普勒效应影响是 一致的,即各阵元接收数据的多普勒因子相同。以 接收阵列的第 $m$ 个通道的接收数据 $\boldsymbol{x}_{m}(t)$ 进行多普 勒因子估计。 $\boldsymbol{x}_{m}(t)$ 和同步信号 $\boldsymbol{s}_{\mathrm{lfm}}(t)$ 做相关检 测, 即有

$$
\boldsymbol{C}_{m}(\tau)=\int_{0}^{\mathrm{T}} \boldsymbol{x}_{m}\left(t_{0}+\tau\right) \boldsymbol{s}_{\mathrm{lfm}}^{*}\left(t_{0}\right) \mathrm{d} t_{0}
$$

相关峰包络 | $\boldsymbol{C}_{m}(\tau) \mid$ 最大值对应的 $\tau$ 为信号从发 射到接收的时间差,根据 $\tau$ 判断回波到达时刻,截取 $\boldsymbol{x}_{m}(t)$ 中的单频信号部分 $\boldsymbol{x}_{m_{-} \mathrm{cw}}(t)$, 以采样频率 $f_{s}$ 得 到序列 $\boldsymbol{x}_{m_{-} \mathrm{cw}}(n)(0 \leqslant n \leqslant N-1)$, 对其做 $N$ 点离散 傅里叶变换 $(\mathrm{DFT})$ 有

$$
\boldsymbol{F}_{m_{-\mathrm{cw}}}(k)=\operatorname{DFT}\left[\boldsymbol{x}_{m_{-\mathrm{cw}}}(n)\right]=\sum_{n=0}^{N-1} \boldsymbol{x}_{m_{-} \mathrm{cw}}(n) \boldsymbol{W}_{N}^{k n}
$$

式中: $\boldsymbol{W}_{N}=\mathrm{e}^{-\mathrm{j} 2 \pi / N}$, 频谱幅值 $\left|\boldsymbol{F}_{m_{-\mathrm{cw}}}(k)\right|$ 最大位置 对应的频率为 $f_{\mathrm{dft}}$, 即有多普勒频偏为

$$
\Delta_{\mathrm{dft}}=f_{\mathrm{dft}}-f_{0}
$$

由此可得多普勒因子估计值 $\hat{\alpha}_{\mathrm{dft}}$ 为

$$
\hat{\alpha}_{\mathrm{dft}}=\frac{\Delta_{\mathrm{dft}}}{f_{0}}
$$

利用 DFT 求多普勒因子,其精度受限于 DFT 频 谱分辨率 $\Delta f=f_{s} / N$, 频率最大误差为 $\pm 0.5 \Delta f$, 因此 多普勒因子估计存在较大误差。为了增加频谱分辨 率, 又不增加 DFT 计算长度 $N$, 利用 CZT 对 $f_{\mathrm{dft}}$ 附近 频带 $\left[f_{\mathrm{dft}}-\kappa \cdot \Delta f, f_{\mathrm{dft}}+\kappa \cdot \Delta f\right]$ 进行细化。由于频率 最大误差为 $\pm 0.5 \Delta f, \kappa$ 一般取大于 1 的整数, 同时为 了保证在 $f_{\mathrm{dft}}$ 附近, $\kappa$ 取值不宜大于 5 。即有频谱细
化表达式为

$$
\begin{gathered}
\boldsymbol{X}_{\mathrm{czt}}\left(z_{k}\right)=\mathrm{CZT}\left[\boldsymbol{x}_{m_{-} \mathrm{cw}}(n)\right]= \\
\sum_{n=0}^{N-1} \boldsymbol{x}_{m_{-} \mathrm{cw}}(n) z_{k}^{-n}= \\
\sum_{n=0}^{N-1} \boldsymbol{x}_{m_{-} \mathrm{cw}}(n) A^{-n} W^{-n k}
\end{gathered}
$$

式中: $A=A_{0} \mathrm{e}^{\mathrm{j} \phi_{0}}, W=W_{0} \mathrm{e}^{-\mathrm{j} \phi_{\Delta}}, A_{0}$ 与 $W_{0}$ 为任意正实 数; $\phi_{0}$ 为起始幅角; $\phi_{\Delta}$ 为幅角增量, $k=0,1, \cdots, K-$ 1 。随着 $k$ 的变化, CZT 在 $Z$ 平面上的变化路径是一 条螺旋线。为对信号进行谱分析, 在单位圆上实现 $\mathrm{CZT}$, 取 $A_{0}=1, W_{0}=1$ 。为了简化计算, 将 $n k=\left[n^{2}+\right.$ $\left.k^{2}-(k-n)^{2}\right] / 2$ 代人(14) 式中, 有

$$
\boldsymbol{X}_{\text {czt }}\left(z_{k}\right)=W^{\frac{k^{2}}{2}} \sum_{n=0}^{N-1} \boldsymbol{x}_{m_{-} \mathrm{cw}}(n) A^{-n} W^{\frac{n^{2}}{2}} W^{\frac{-(n-k)^{2}}{2}}
$$

式中, $\phi_{0}=\mathrm{e}^{\mathrm{j} 2 \pi\left(f_{\mathrm{dft}}-\kappa \cdot \Delta f\right) / f_{s}}, \phi_{\Delta}=\mathrm{e}^{-\mathrm{j} 2 \pi \cdot 2 \kappa \cdot \Delta f /\left(f_{s} K\right)}$ 。将采样 点 $z_{k}$ 的 $\mathrm{Z}$ 变换表示为 $\boldsymbol{g}(k)$ 与 $\boldsymbol{h}(k)$ 的线性卷积与 $W^{k^{2 / 2}}$ 的乘积, 即

$$
\begin{gathered}
\boldsymbol{X}_{\mathrm{czt}}\left(z_{k}\right)=W^{k^{2} / 2} \sum_{n=0}^{N-1} \boldsymbol{g}(n) \boldsymbol{h}(k-n)= \\
W^{k^{2} / 2}[\boldsymbol{g}(k) * \boldsymbol{h}(k)]
\end{gathered}
$$

式中, $\boldsymbol{g}(k)=\boldsymbol{x}_{m_{-\mathrm{cw}}}(k) A^{-k} W^{k^{2 / 2}}, \boldsymbol{h}(k)=W^{-k^{2 / 2}}$ 。当信 号序列长度为 $N$, 频谱分析点数为 $K, \boldsymbol{g}(k) * \boldsymbol{h}(k)$ 圆 周卷积的点数为 $P \geqslant K+N-1$, 为了便 FFT 运算, $P$ 满足 2 的最小幂次方。因此, CZT 细化的计算杂度 为 $3 P \log _{2} P / 2+5 N+P+K_{\circ}$ 信号为单频率成分情况 下,CZT 理论上可以无限细化频率分辨率,但无限细 化会增加算法计算复杂度。

\section{2 频谱校正多普勒精估计与补偿}

为了降低 CZT 频谱细化增加的计算复杂度而 不影响估计精度,在减少频谱细化倍数的同时利用 频谱校正进行弥补。对接收数据加 Hanning 窗采 样, Hanning 窗定义为

$$
W(n)=0.5-0.5 \cos (2 \pi n / N)
$$

窗长点数为 $N$,其归一化频谱模函数为

$$
W_{1}(f)=\frac{\sin (\pi f)}{\pi f} \cdot \frac{1}{2\left(1-f^{2}\right)}
$$

对于幅值为 $B$, 频率为 $f_{0}$ 的正弦信号加 Hanning 窗, 则其频谱主瓣内的模函数的平方为

$$
G_{f}=B \frac{\sin ^{2}\left[\pi\left(f-f_{0}^{1}\right)\right]}{4 \pi^{2}\left(f-f_{0}^{1}\right)^{2}\left[1-\left(f-f_{0}^{1}\right)^{2}\right]^{2}}
$$

式中: $f_{0}^{1}$ 为主瓣中心估计值; $G_{q}$ 为功率谱第 $q$ 条谱线 值; $G_{Q}$ 为主瓣内谱线最大值; $Q$ 为对应的谱线。根 
据 Hanning 窗的特性, 当 $p \rightarrow \infty$ 有

$$
\begin{gathered}
\sum_{q=-p}^{p} G_{Q+q}\left(Q-f_{0}^{1}+q\right)= \\
\sum_{q=-p}^{p} G_{Q+q}(Q+q)-f_{0}^{1} \sum_{q=-p}^{p} G_{Q+q}=0
\end{gathered}
$$

即可求得主瓣中心估计值 $f_{0}^{1}$ 为

$$
f_{0}^{1}=\frac{\sum_{q=-p}^{p}(Q+q) G_{Q+q}}{\sum_{q=-p}^{p} G_{Q+q}}
$$

根据(16) 式进行 CZT 频谱细化后, 频率分辨率变为 $\Delta f_{\text {czt }}=2 \kappa \cdot \Delta f / M$, 即有频谱细化与校正联合估计的 频率 $\hat{f}_{0}$ 为

$$
\hat{f}_{0}=f_{0}^{1} \Delta f_{\text {czt }}=\frac{\sum_{q=-p}^{p}(Q+q) G_{Q+q}}{\sum_{q=-p}^{p} G_{Q+q}} \cdot \frac{2 \kappa \cdot \Delta f}{M}
$$

则多普勒频偏 $\Delta=\hat{f}_{0}-f_{0}$, 由此可得多普勒因子 $\alpha$ 为

$$
\hat{\alpha}=\frac{\Delta}{f_{0}}
$$

由于频谱校正只需要简单的几次乘法和加法运 算, 其计算复杂度远小于 CZT 的计算量, 因此基于 频谱细化与校正联合多普勒估计方法有效降低计算 复杂度。

根据估计得到的多普勒因子对阵列接收数据 $X(t)$ 进行多普勒补偿, 其补偿后的数据为

$$
\boldsymbol{X}_{d c}(t)=\boldsymbol{X}((1-\hat{\alpha}) t)
$$

对补偿后的 $\boldsymbol{X}_{d c}(t)$ 利用 (8) 式与 (9) 式进行目标 DOA 估计, 能有效降低多普勒效应对主动探测目标 参数估计性能的不利影响。

\section{3 所提算法流程}

基于通信信号的水下目标主动探测多普勒估计 与补偿算法如下所示:

算法:基于频谱细化与校正的联合多普勒估计与补 偿方法

输人: 接收信号 $\boldsymbol{x}_{m}(t)$, 同步头副本信号 $\boldsymbol{s}_{\mathrm{lfm}}(t), \kappa$; 输出: 多普勒因子估计值 $\hat{\alpha}$, 目标方位角 $\theta$;

1. $\boldsymbol{x}_{m}(t)$ 与 $\boldsymbol{s}_{\mathrm{lfm}}(t)$ 作互相关, 获取单频信号 段 $\boldsymbol{x}_{m_{-} \mathrm{cw}}(t)$;

2. 对 $\boldsymbol{x}_{m_{-} \mathrm{cw}}(t)$ 加 Hanning 窗, 并作 $N$ 点 DFT 求得最 大幅值对应频率 $f_{\mathrm{dft}}$;

3. 利用 (17) 式对频率 $f_{\mathrm{dft}}$ 附近的频谱范围 $\left[f_{\mathrm{dft}}-\kappa \cdot\right.$
$\left.\Delta f, f_{\mathrm{dft}}+\kappa \cdot \Delta f\right]$ 进行频谱细化;

4. 利用(23) 式对细化后频谱进行校正, 得到校正后 最大幅值对应的频率 $\hat{f}_{0}$;

5. 根据 (23) 式求得 $\hat{\alpha}$, 根据 $\hat{\alpha}$ 利用(24) 式对阵列接 收信号 $\boldsymbol{X}(t)$ 进行多普勒补偿得到 $\boldsymbol{X}_{d c}(t)$;

6. 对补偿的接收信号 $\boldsymbol{X}_{d c}(t)$ 利用 (8) 式与 (9) 式求 目标方位角 $\theta$ 。

\section{3 仿真实验与性能分析}

本节仿真分析多普勒效应对 DOA 估计影响、所 提算法的多普勒因子估计性能以及多普勒后的 DOA 估计性能。发射的共享信号中, LFM 同步信号 脉宽 $0.1 \mathrm{~s}$, 频带 $5.5 \sim 6.5 \mathrm{kHz}$; 单频信号为正弦信 号, 频率为 $10 \mathrm{kHz}$, 脉宽 $0.1 \mathrm{~s}$; 通信信号为 BPSK 调 制信号, 信号带宽 $4 \sim 8 \mathrm{kHz}$, 载频率为 $f_{c}=6 \mathrm{kHz}_{\circ}$ 信 号采样频率为 $f_{s}=48 \mathrm{kHz}$, 接收阵为八元均匀线列 阵, 阵元间距为通信信息信号段 $s_{\mathrm{com}}(t)$ 载频率对应 的半波长。

\section{1 多普勒效应对 DOA 估计性能影响}

当多普勒因子 $\alpha$ 分别为 $0, \pm 0.005$ 时,采用互相 关 MUSIC 算法分别进行单目标与双目标 DOA 估 计。对于单目标, 目标人射角为 $14^{\circ}$ 。对于双目标, 人射角度分别为 $\left[-5^{\circ} 5^{\circ}\right]$ 。利用均方误差 $\left(E_{\mathrm{MS}}\right)$ 以 及分辨概率对估计性能进行评估, $E_{\mathrm{MS}}$ 定义为

$$
E_{\mathrm{MS}}=\frac{1}{J} \sum_{j=1}^{J}\left(\hat{\theta}_{k j}-\theta_{k}\right)^{2}
$$

式中, $J$ 为蒙特卡洛实验次数, $\hat{\theta}_{k j}$ 为第 $j$ 次蒙特卡洛 实验对目标方位角 $\theta_{k}$ 的估计值。目标可分辨满足

$\max \left\{\left|\hat{\theta}_{1}-\theta_{1}\right|,\left|\hat{\theta}_{2}-\theta_{2}\right|, \cdots,\left|\hat{\theta}_{K}-\theta_{K}\right|\right\} \leqslant \frac{W_{B}}{2}$

式中, $W_{B}$ 为波束宽度。在信噪比 $R_{\mathrm{SN}}$ 从- $24 \mathrm{~dB}$ 到 $4 \mathrm{~dB}$ 以 $2 \mathrm{~dB}$ 号进增加, 分别进行 1000 次蒙特卡洛 仿真实验,其仿真结果如图 2 所示。

由图 2 可知, 在单目标与双目标估计中, 多普勒 因子 $\alpha=0$ 时的 DOA 估计性能优于多普勒因子 $\alpha=$ \pm 0.005 时的 DOA 估计性能, 说明多普勒效应降低了 互相关 MUSIC 算法的 DOA 估计性能。这是由于多 普勒效应降低了发射副本与接收信号互相关处理增 益,进而降低了DOA 估计性能。 


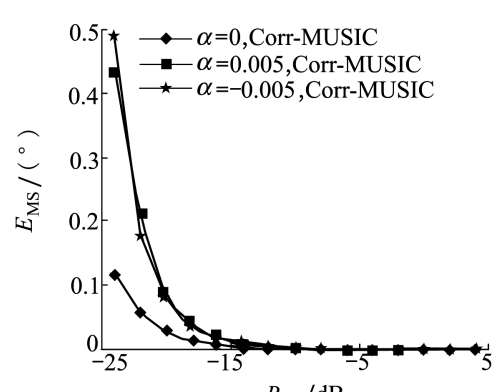

a) 单目标方均误差

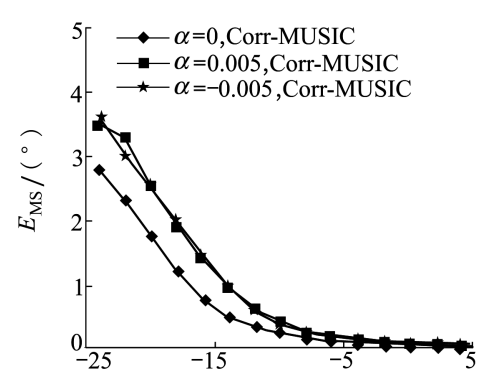

b) 双目标方均误差

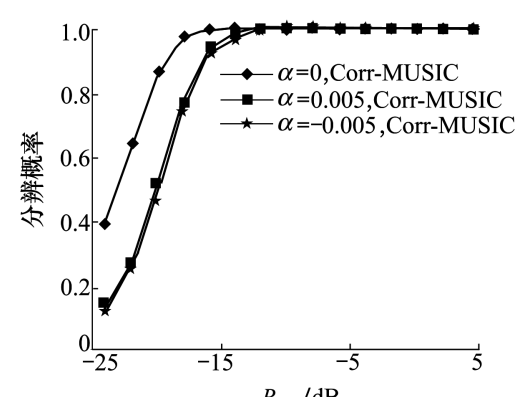

c) 单目标分辩概率

图 2 多普勒效应对探测性能的影响

\section{2 多普勒估计性能}

仿真比较经典 FFT、FFT 抛物线插值、CZT 频谱 细化、能量频谱校正以及本文所提方法多普勒估计
精度。在多普勒因子分别为 $0.006,0.01$ 以及 0.032 , 信噪比 $R_{S N}=-10 \mathrm{~dB}$ 时,不同算法频率估计值如图 3 所示。

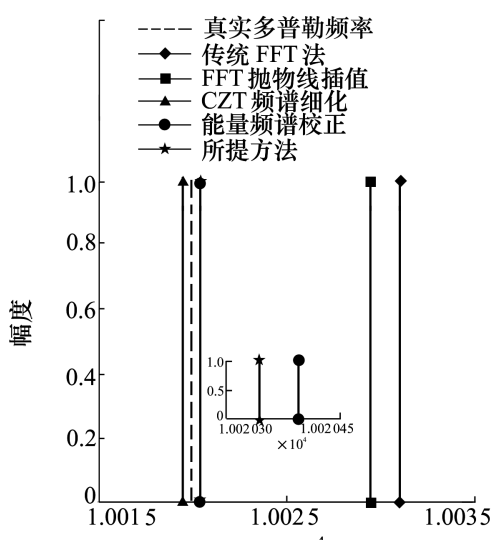

频率 $/ 10^{4} \mathrm{~Hz}$ a) $\alpha=0.006$

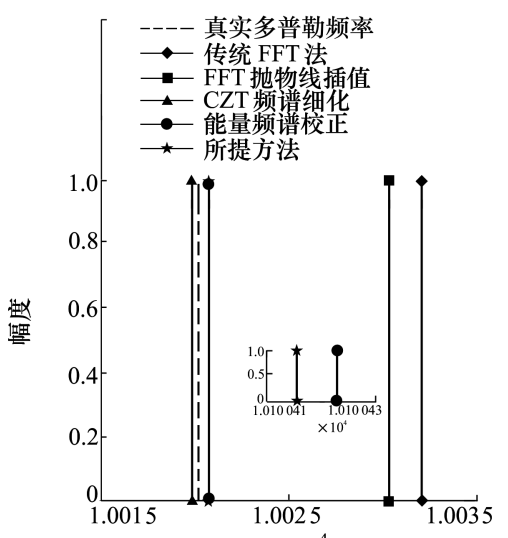

频率 $/ 10^{4} \mathrm{~Hz}$

b) $\alpha=0.01$

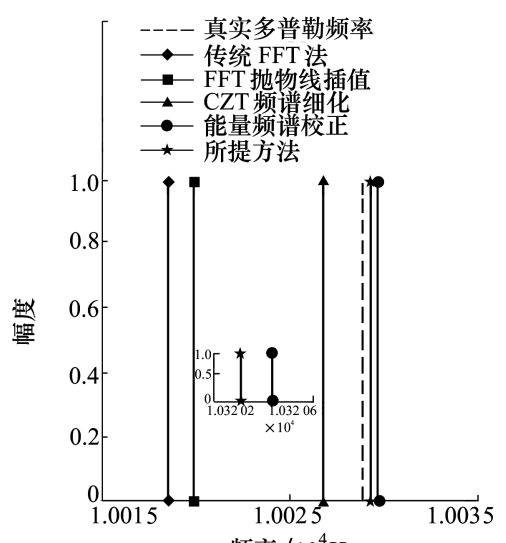

频率 $/ 10^{4} \mathrm{~Hz}$ c) $\alpha=0.032$

图 $3 R_{S N}=-10 \mathrm{~dB}$ 时多普勒因子估计

从图 3 可以看出, 采用传统 FFT 估计的频率距 离真实多普勒频率最远, 误差最大。经过抛物线插 值后, 一定程度上提高了频率估计精度。并且在所 比较的方法中, 所提方法估计的频率与真实多普勒 频率最接近, 估计误差最小。因此, 所提方法对频率
估计具有较高的精度。

在信噪比 $R_{\mathrm{SN}}$ 从- $18 \mathrm{~dB}$ 到 $6 \mathrm{~dB}$ 以步进 $2 \mathrm{~dB}$ 增 加, 分别仿真不同算法在多普勒因子为 $0.006,0.01$ 以及 0.032 情况下的多普勒估计性能, 分别进行 1000 次蒙特卡洛仿真实验, 仿真结果如图 4 所示。

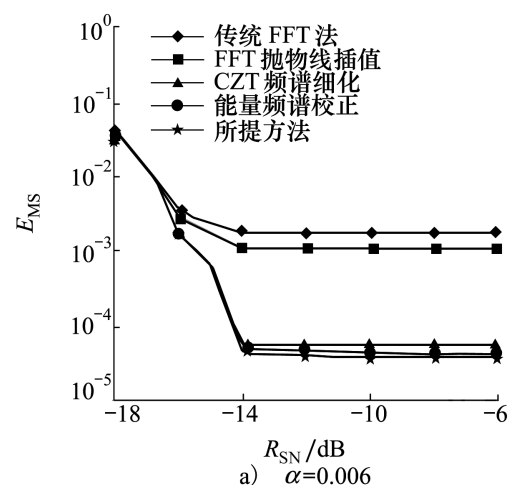

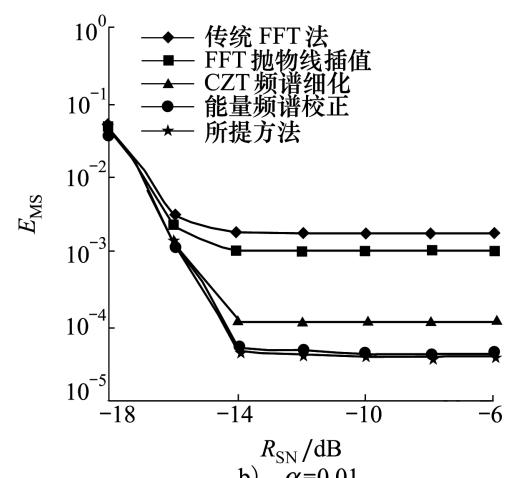

b) $\quad \alpha=0.01$

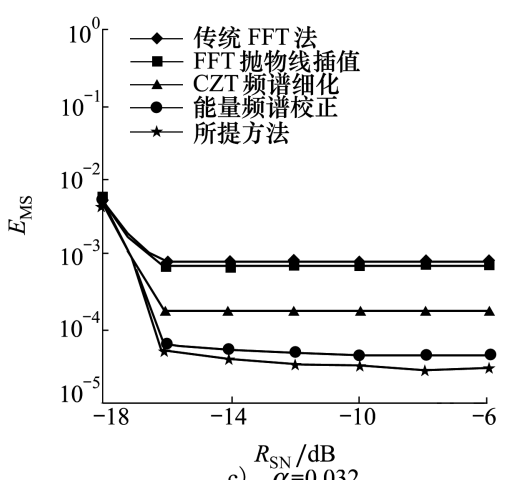

c) $\alpha=0.032$

图 4 不同信噪比下多普勒因子估计 
从图 4 中可以看出, 所提方法在不同多普勒因 子下均具有最优的多普勒估计精度, 能量谱校正方 法次之。传统 FFT 法最差, 而抛物线插值在一定程 度上可以提高 FFT 方法估计精度。
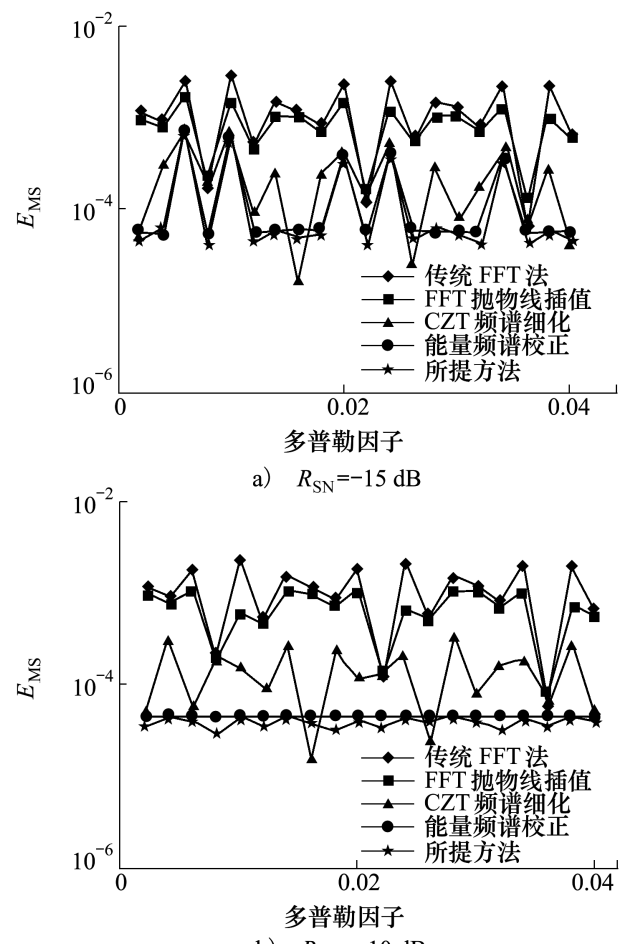

b) $R_{\mathrm{SN}}=-10 \mathrm{~dB}$
当多普勒因子为 $\alpha$ 从 0.002 到 0.04 , 以步进 0.002 增加, 仿真信噪比为 $-15 \mathrm{~dB}$ 以及 $-10 \mathrm{~dB}$ 情况 下不同算法的多普勒估计性能,分别进行 1000 次 蒙特卡洛仿真实验,仿真结果如图 5 所示。

从图 5 可以看出,在不同的多普勒因子与信噪 比下, 所提方法具有最优的估计性能, 且对于不同的 多普勒因子具有稳定的估计性能。

\section{3 多普勒补偿与参数估计}

利用估计的多普勒因子对阵列接收信号进行多 普勒补偿, 并利用互相关 MUSIC 算法对补偿后的阵 列接收信号进行 DOA 参数估计。对于单目标, 目标 人射角为 $14^{\circ}$ 。对于双目标, 目标人射角分别为 $\left[-4^{\circ} 4^{\circ}\right]$ 。在不同信噪比 $R_{\mathrm{SN}}$ 为 $18 \mathrm{~dB}$ 到 $6 \mathrm{~dB}$, 以 步进 $2 \mathrm{~dB}$ 增加, 分别仿真多普勒因子为 $0.006,0.01$ 以及 0.032 情况下, 不同算法多普勒估计与补偿对 DOA 估计性能的影响, 分别进行 1000 次蒙特卡洛 仿真实验。

图 6 给出单个目标情况下, 利用不同多普勒估 计方法进行多普勒估计, 并对阵列接收信号进行多 普勒补偿, 然后采用互相关 MUSIC 算法进行 DOA 参数估计。从图中可以看出对不同多普勒因子, 利 用所提方法估计的多普勒, 信多普勒补偿后 DOA 估 计性能最优。

图 5 不同多普勒因子估计下多普勒估计

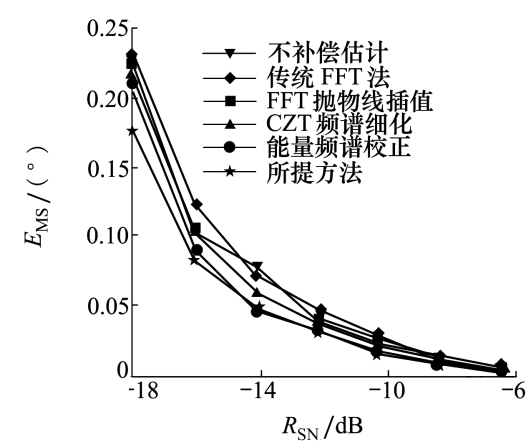

a) $\alpha=0.006$

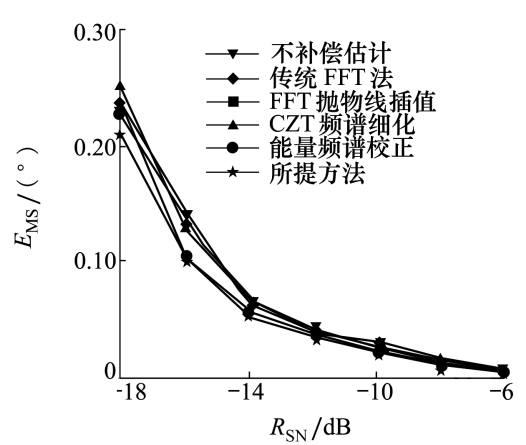

b) $\alpha=0.01$

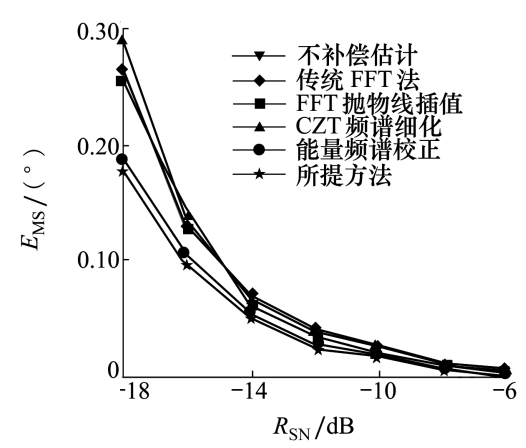

c) $\alpha=0.032$

图 6 不同信噪比下单目标 DOA 估计精度

图 7 至 9 给出不同多普勒效应下的双目标估计 性能。利用不同多普勒估计方法估计出多普勒因 子, 对接收信号进行多普勒补偿后, 采用互相关 MUSIC 算法得到 DOA 估计。从图 7 至图 9 可以看出, 在不同的多普勒因子下, 利用所提方法估计的多普 勒因子, 对接收信号补偿后的 DOA 估计具有具最小 的估计误差以及最优的分辨概率, 能量频谱校正法
次之,均优于未补偿的。

从上面的仿真结果还可以看出未进行多普勒补 偿的 DOA 估计性能不一定是最差的, 因为当多普勒 估计精度不高时, 多普勒补偿不但不能提高 DOA 估 计性能, 反而会引人误差降低估计性能。因此, 在进 行多普勒补偿时需要在一定的多普勒估计精度下进 行, 否则会引人误差降低参数估计精度。 


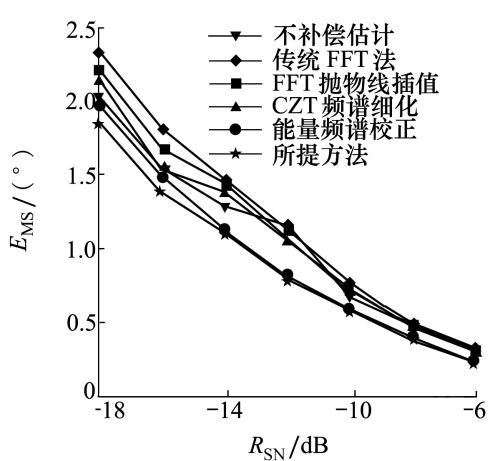

a) 双目标 DOA 估计均方误差

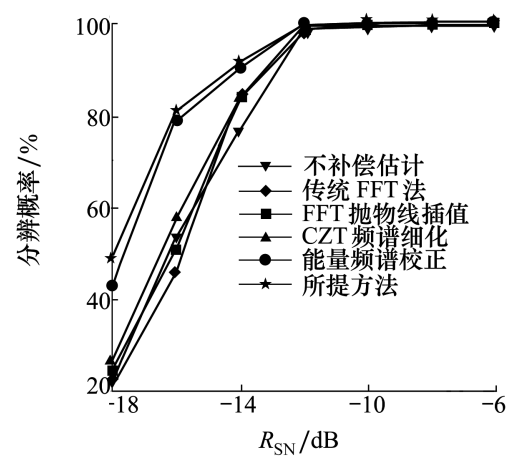

b） 分辩概率与信噪比 $R_{\mathrm{SN}}$ 关系

图 $7 \alpha=0.006$ 时双目标估计性能

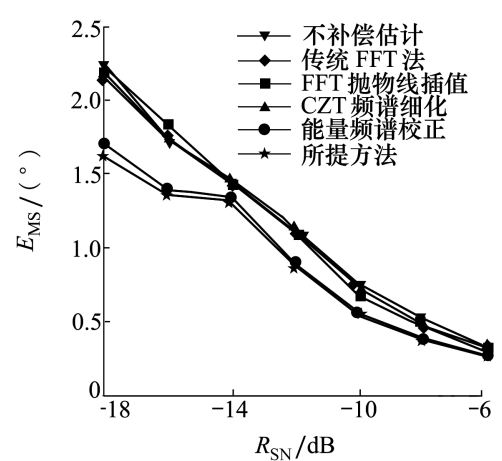

a) 双目标 DOA 估计均方误差

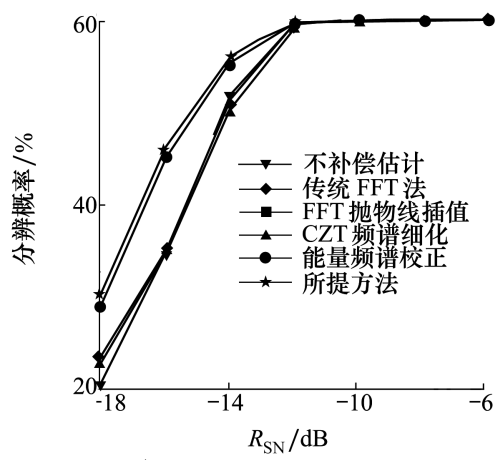

b）分辩概率与信噪比 $R_{\mathrm{SN}}$ 关系

图 $8 \alpha=0.01$ 时双目标估计性能

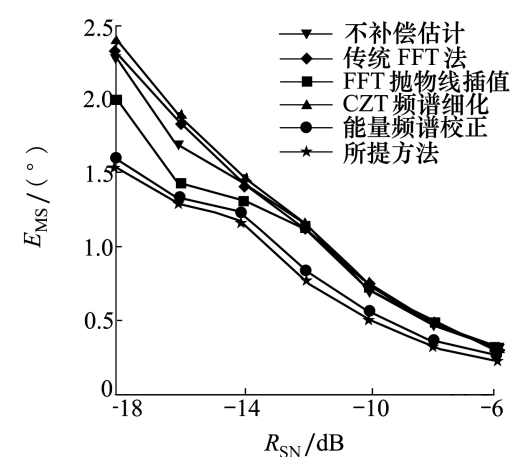

a) 双目标 DOA 估计均方误差

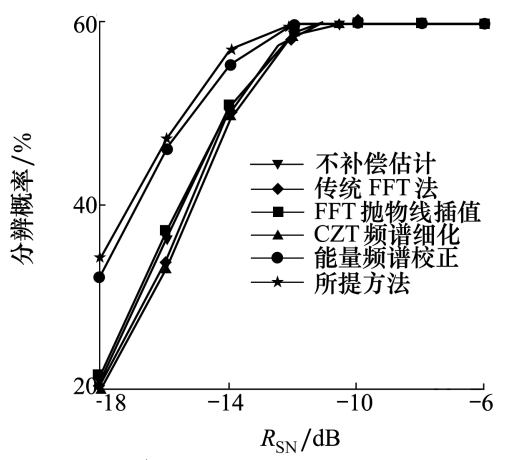

b） 分辩概率与信噪比 $R_{\mathrm{SN}}$ 关系

图 $9 \alpha=0.032$ 时双目标估计性能

\section{4 结 论}

针对多普勒效应对水下探测通信一体化系统中 目标主动探测参数估计性能恶化的问题, 利用对阵 列接收信号进行多普勒补偿, 减小或消除多普勒效 应的影响, 提出基于频谱细化与校正的多普勒估计 与补偿方法。首先利用同步信号获取回波时延, 获
取回波中单频信号段。然后采用频谱细化与校正进 行联合多普勒估计，得到多普勒因子。同时对阵列 接收信号进行多普勒补偿, 并利用互相关 MUSIC 算 法进行 DOA 估计。仿真结果表明,所提算法能提高 多普勒估计精度, 并且对不同的多普勒具有良好鲁 棒性。同时,多普勒估计性能的提高使得多普勒补 偿更为准确, 从而增加了互相关处理增益, 提高了 DOA 估计精度。

\section{参考文献:}

[1] 卢俊,张群飞,史文涛. 水下探测通信一体化关键技术分析 [J]. 水下无人系统学报,2018, 26(5): 106-115

LU Jun, ZHANG Qunfei, SHI Wentao. Analysis on the key technology of integrated underwater detection and communication [J]. Journal of Unmanned Undersea Systems, 2018, 26(5) : 470-479 (in Chinese)

[2] KASSEM J, BARBEAU M, et al. Doppler effect in the acoustic ultra low frequency band for wireless underwater networks $[\mathrm{J}]$. Mobile Networks and Applications, 2018, 23: 1282-1292

[3] ZHOU J, NIE X M, LIN J. A novel laser doppler velocimeter and its integrated navigation system with strapdown inertial navigation [J]. Optics \& Laser Technology, 2014, 64: 319-323

[4] 厉文涛, 聂晓明, 周健. 基于二维激光多普勒测速仪建立新组合导航系统的方法 [ J]. 中国激光, 2020, 47(3) : 1-6 LI Wentao, NIE Xiaoming, ZHOU Jian. Method for establishing new integrated navigation system based on two-dimensional laser doppler velocimete $[\mathrm{J}]$. Chinese Journal of Lasers, 2020, 47(3): 1-6 (in Chinese)

[5] DENBIGH P N. Ship velocity determination by doppler and correlation techniques [J]. IEEE Proceeding F-Commanications, Radar and Signal Processing, 1984, 131(3) : 315-326 
[6] ZEDEL L, HAY A E. Resolving velocity ambiguity in multi-frequency, pulse-to-pulse coherent doppler sonar[ J]. IEEE Journal of Oceanic Engineering, 2010, 35(4): 847-851

[7] WANIS P, BRUMLEY B, GAST J, et al. Sources of measurement variance in broadband acoustic doppler current profilers [ C] // OCEANS 2010 MTS/IEEE SEATTLE, Seattle, WA, 2010: 1-5

[8] CHEN Y, YIN J, ZOU L. Null subcarriers based doppler scale estimation with polynomial interpolation for multicarrier communication over ultrawideband underwater acoustic channels [ J ]. Journal of Systems Engineering \& Electronics, 2015 ( 6) : 1177-1183

[9] ABDELKAREEM A E, SHARIF B S, TSIMENIDIS C C, et al. Time varying doppler-shift compensation for OFDM-based shallow underwater acoustic communication systems[C]//IEEE International Conference on Mobile Adhoc \& Sensor Systems, 2011

[10] TANG C, LIAN B, ZHANG L. Doppler-aided channel estimation in satellite communication base on frequency-domain equalization [C] // 2013 Integrated Communications, Navigation and Surveillance Conference, 2013

[11] YILMAZ U C, CAVDAR I H. The effects of orbital parameters accuracy on doppler frequency shift for intersatellite optical communication [C] //International Conference on Application of Information \& Communication Technologies, 2013

[12] SEN S, NEHORAI A. Adaptive design of OFDM radar signal with improved wideband ambiguity function [J]. IEEE Trans on Signal Processing, 2010, 58(2) : 928-933

[13] RIFE D C, VINCENT G A. Use of the discrete fourier transform in the measurement of frequencies and levels of tones[ $\mathrm{J}]$. Bell Labs Technical Journal, 1970, 49(2): 197-228

[14] 齐国清, 贾欣乐. 插值 FFT 估计正弦信号频率的精度分析 $[\mathrm{J}]$. 电子学报, 2004, 32(4):625-629

QI Guoqing, JIA Xinle. Accuracy analysis of frequency estimation of sinusoid based on interpolate FFT[J]. Acta Electronica Sinica, 2004, 32(4) : 625-629 (in Chinese)

[15] SONG M, LIM J, SHIN D, et al. Enhancing doppler estimation via newton interpolation for automotive FMCW radars [C] //2014 International Conference on Information and Communication Technology Convergence, Busan, 2014: 615-616

[16] 陆松鹤. 多载波移动水声通信中的同步技术研究 [D]. 哈尔滨: 哈尔滨工程大学, 2009

LU Songhe. Study on synchronization in multi-carrier mobile underwater acoustic communication [D]. Harbin: Harbin Engineering University, 2009 (in Chinese)

[17] 赵宏强. 频谱细化算法分析 $[\mathrm{J}]$. 四川兵工学报, 2013, 34(5) : 105-112

ZHAO Hongqiang. Analysis of spectrum zoom algorithms[ J]. Journal of Ordnance Equipment Engineering, 2013, 34( 5) : 105112 (in Chinese)

[18] SYSEL P, RAJMIC P. Goertzel algorithm generalized to non-integer multiples of fundamental frequency [J]. Journal on Advances in Signal Processing, 2012, 56: 1-8

[19] MA L, JIA H, LIU S, et al. Low-complexity doppler compensation algorithm for underwater acoustic OFDM systems with nonuniform doppler shifts [J]. IEEE Communications Letters, 2020, 24(9) : 2051-2054

[20］宋金金超，刘启明，苏庆堂. 基于 FFT 最大谱线处相位差的频谱校正方法 [J]. 控制工程，2017，24(5):1086-1089 SONG Xinchao, LIU Qiming, SU Qingtang. Spectrum correction method based on FFT maximum spectrum phase difference[J]. Contral Engineering of China, 2017, 24( 5) : 1086-1089 (in Chinese)

[21] HUANG R, DU X Y, HU W D. UAV target detection and parameter estimation in non-homogeneous clutter[J]. The Journal of Engineering, 2019, 20: 6750-6754

[22] 丁康, 谢明, 杨志坚. 离散频谱分析校正理论与技术 [M]. 北京: 科学出版社, 2008

DING Kang, XIE Ming, YANG Zhijian. The theory and technology of discrete spectrum correction[M]. Beijing: Beijing Science Press, 2008 (in Chinese)

[23] 朱小勇,丁康. 离散频谱校正方法的综合比较 $[\mathrm{J}]$. 信号处理, 2001,17( 1) : 91-97

ZHU Xiaoyong, DING Kang. Comprehensive comparison of discrete spectrum correction methods [J]. Signal Processing, 2001, 17( 1) : 91-97 (in Chinese) 


\title{
Doppler estimation and compensation method for underwater target active detection based on communication signal
}

\author{
LU Jun, ZHANG Qunfei, SHI Wentao, ZHANG Lingling
}

( School of Marine Science and Technology, Northwestern Polytechnical University, Xi' an 710072, China)

\begin{abstract}
The integration of underwater detection and communication uses communication signals to detect a target actively, but the Doppler effect deteriorates the parameter estimation performance of the integrated system. To eliminate the influence of the Doppler effect, a joint Doppler estimation and compensation method based on spectrum zooming and correction is proposed. Firstly, the synchronization signal is used to obtain the signal receiving delay and intercept the single-frequency signal segment in the received signal. Then, the discrete Fourier transform is used to find the frequency that corresponds to the maximum amplitude of the single-frequency signal segment. Finally, the frequency spectrum is refined and corrected within the range near the frequency. The Doppler factor is estimated and the received signal is compensated by the Doppler estimation value. The simulation results show that the proposed method improves Doppler factor estimation accuracy, increases the cross-correlation processing gain and improves DOA ( direction of arrival) estimation performance, thus being robust to different Doppler effects.
\end{abstract}

Keywords: Doppler estimation and compensation; integration of underwater detection and communication; communication signal; spectrum zooming; spectrum correction

引用格式:卢俊, 张群飞, 史文涛, 等. 基于通信信号的水下目标主动探测多普勒估计与补偿方法 $[J]$. 西北工业大学学报, $2021,39(5): 962-970$

LU Jun, ZHANG Qunfei, SHI Wentao, et al. Doppler estimation and compensation method for underwater target active detection based on communication signal[J]. Journal of Northwestern Polytechnical University, 2021, 39(5): 962-970 (in Chinese) 